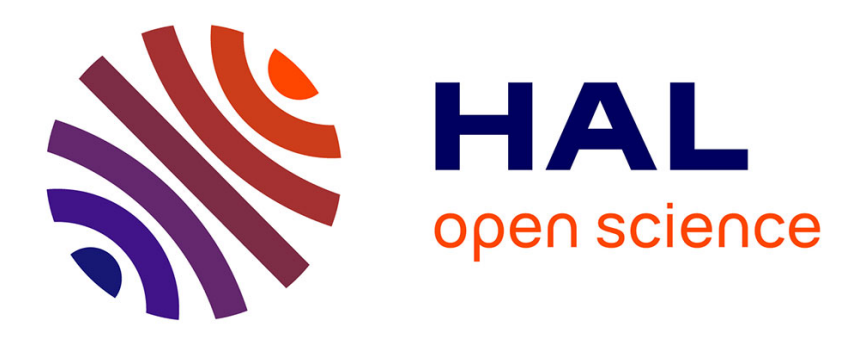

\title{
Modélisation impulsionnelle de l'interaction entre un faisceau ultrasonore et un défaut proche de la paroi externe
}

\author{
O. Roy, P. Calmon
}

\section{- To cite this version:}

O. Roy, P. Calmon. Modélisation impulsionnelle de l'interaction entre un faisceau ultrasonore et un défaut proche de la paroi externe. Journal de Physique IV Proceedings, 1994, 04 (C5), pp.C5-1169C5-1172. 10.1051/jp4:19945257 . jpa-00252946

\section{HAL Id: jpa-00252946 https://hal.science/jpa-00252946}

Submitted on 1 Jan 1994

HAL is a multi-disciplinary open access archive for the deposit and dissemination of scientific research documents, whether they are published or not. The documents may come from teaching and research institutions in France or abroad, or from public or private research centers.
L'archive ouverte pluridisciplinaire HAL, est destinée au dépôt et à la diffusion de documents scientifiques de niveau recherche, publiés ou non, émanant des établissements d'enseignement et de recherche français ou étrangers, des laboratoires publics ou privés. 


\title{
Modélisation impulsionnelle de l'interaction entre un faisceau ultrasonore et un défaut proche de la paroi externe
}

\author{
O. ROY et P. CALMON
}

CEN Saclay, DTM/STA/LCUS, Bâtiment C111, 91191 Gif sur Yvette, France

\begin{abstract}
In non destructive testing, the corner effect is the result of the interaction between an ultrasonic focused beam and a plane defect located near the opposite surface of the controlled block. This paper presents a model developed in order to predict corner effect echoes. Its principle is based on the computation of the spatial impulse response for both transmitting and receiving mode. Some results are presented and compared to experiment.
\end{abstract}

\section{INTRODUCTION}

Lorsqu'une pièce contient un défaut situé à proximité de la surface opposée au contrôle, on détecte par ultrasons un ou plusieurs échos importants dits échos de coin. Ces échos sont dus à la réflexion successive du faisceau incident sur le défaut et le fond de la pièce. Nous présentons un modèle dont l'objet est la prédiction des résultats concernant ces échos: amplitude, temps de vol et forme d'onde en fonction du balayage. On modélise une pièce homogène et isotrope, contrôlée en immersion avec un traducteur fonctionnant en émission-réception. Le modèle est basé sur le calcul de la réponse impulsionnelle du traducteur après réfraction sur une interface plane liquide-solide. L'interaction avec le défaut est traitée dans une approximation de type Kirchhoff, et la réception est calculée en appliquant un principe de réciprocité entre émission et réception.

\section{LA REPONSE IMPULSIONNELAE DE DIFFRACTION}

En théorie classique de l'acoustique linéaire, la pression instantanée, engendrée en un point $M$ de l'espace par un transducteur placé dans un milieu homogène et isotrope, est proportionnelle à la dérivée temporelle du potentiel de vitesse instantané en ce point. L'intégrale de Rayleigh, sous sa forme temporelle, permet de calculer le potentiel de vitesse en un point $M$ et au temps $t$, pour une ouverture diffractante connue. Le potentiel de vitesse s'exprime alors comme la somme au point $M$ des contributions de chaque élément de surface:

$$
\phi(M, t)=\int_{\text {traducteur }} \frac{v(t-r / c)}{2 \pi r} d s=v_{0}(t) \otimes \int_{\text {traducteur }} \frac{\delta(t-r / c)}{2 \pi r} d s=v_{0}(t) \otimes r(M, t)
$$

où $v$ est la vitesse normale à la surface émettrice, $r$ est la distance reliant un élément de surface émettrice au point $M$, et $c$ est la vitesse de propagation des ondes dans le milieu. Pour une vitesse normale à la surface uniforme, le potentiel de vitesse peut s'exprimer comme le produit de convolution du signal d'excitation, $v_{0}(t)$, et de la réponse impulsionnelle de diffraction au point $M, r(M, t)$. Cette réponse est donc équivalente au potentiel de vitesse obtenu pour un signal d'excitation infiniment bref. Cette formulation du potentiel de vitesse instantanée peut être étendue au cas de deux milieux de propagation séparé par une interface infinie dans l'approximation de l'optique géométrique. Cette approximation est valable si le point d'observation est suffisamment éloigné de l'interface [1]. On définit alors la réponse impulsionnelle par: 
$\phi(M, t)=v_{0}(t) \otimes \int_{\text {traducteur }} C\left(\theta_{1}, t\right) \otimes \frac{\delta(t-T)}{2 \pi R} d s=v_{0}(t) \otimes r(M, t)$

avec

$T=\frac{R_{1}}{C_{1}}+\frac{R_{2}}{C_{2}} \quad$ et $\quad R=\sqrt{\left(R_{1}+R_{2} \frac{C_{2}}{C_{1}}\right)\left(R_{1}+R_{2} \frac{C_{2}}{C_{1}} \frac{\cos ^{2} \theta_{1}}{\cos ^{2} \theta_{2}}\right)}$

où $T$ est le temps de parcours le long de chaque trajet acoustique, et $R$ représente la conservation de l'énergie dans un cône issu d'une source sphérique dans le milieu 1 vers le point $M$ dans le milieu 2. $R_{I}, C_{l}$, $R_{2}$ et $C_{2}$ sont respectivement les trajets parcourus et les vitesses de propagations dans les deux milieux. $\theta_{I}$ et $\theta_{2}$ définissent l'angle d'incidence et l'angle de réfraction. La distribution $C\left(\theta_{1}, t\right)$ [2]est le coefficient de transmission pour une onde plane impulsionnelle.

Dans le cas d'une interface liquide solide, ce coefficient est indépendant du temps si l'angle d'incidence est inférieur au premier angle critique. La réponse est calculée de manière numérique, en échantillonnant la surface émettrice. Le calcul est effectué de façon approchée en remplaçant la distribution de Dirac par une porte rectangulaire de largeur $\delta \mathrm{t}$ [3]. Cette approximation revient à moyenner la réponse impulsionnelle sur l'intervalle $\delta \mathrm{t}$.

\section{MODELISATION DE L'INTERACTION FAISCEAU-DEFAUT}

Dans un seul milieu de propagation, la réponse impulsionnelle en émission réception après réflexion sur un réflecteur ponctuel peut s'exprimer comme l'auto-convolution de la réponse impulsionnelle de diffraction au point considéré. Cette formulation découle du principe de réciprocité entre l'émission et la réception énoncé par Freedman [4]. En présence d'une interface séparant deux milieux différents, la réciprocité n'est plus vérifiée puisque le coefficient de transmission diffère suivant le sens de passage de l'interface. Cependant le procédé demeure identique, la réponse échographique est le produit de convolution des réponses impulsionnelles de l'émetteur vers le réflecteur, $r_{e}(M, t)$, et du réflecteur vers le récepteur, $r_{r}(M, t)$. Le signal échographique reçu est obtenu en convoluant la réponse impulsionnelle en émission-réception avec une forme d'onde. L'écho généré par un défaut étendu est calculé en sommant les contributions en chacun de ses points considérés comme autant de sources ponctuelles secondaires. Ce faisant, on se place dans une approximation de type Kirchhoff. Dans cette approximation, le signal échographique s'écrit:

$e(x, t)=\frac{\partial^{2} v_{0}(t)}{\partial t^{2}} \otimes \int_{\text {défaut }}\left[r_{e}(M, t) \otimes r_{r}\left(M^{\prime}, t\right)\right] d s$

où $e(x, t)$ est l'amplitude du signal au temps $t$ pour la position $x$ du traducteur.

Chaque point $M$ sur le défaut est soumis au champ ultrasonore du traducteur. Dans le cas de l'effet de coin, ce champ incident après interaction avec le défaut est réfléchi par le fond en direction du traducteur. Le fond étant assimilé à un réflecteur parfait, tout se passe comme si, dans l'approximation de l'optique géométrique, l'onde était issue en retour, d'un point $M^{\prime}$ 'symétrique de $M$ par rapport au fond (fig. 1).

\section{VALIDATION}

Le traitement de l'interaction faisceau-défaut a déjà été confronté à l'expérience. Dans un premier temps nous avons en effet développé un logiciel de simulation des contrôles ultrasonore basé sur une version simplifiée de l'équation (4) qui évitait le calcul de la réponse impulsionnelle, en supposant que le champ dans le faisceau focalisé peut s'écrire [3]:

$\Phi(M, t)=v_{0}(t-z / c) p(\tau, z)$

où $p(\tau, z)$ est une fonction des coordonnées spatiales de $M(\tau$ mesure l'écart à l'axe focal et $z$ la distance le long de cet axe) qui décrit l'amplitude du champ dans le faisceau. Cette approximation s'appuie sur le fait que les fronts d'onde sont quasiment plans dans la zone focale. Ce modèle simplifié permet de prédire correctement les courbes échodynamiques (amplitude en fonction du balayage), ainsi que les bscans (temps de vol en fonction du balayage). La figure (2) montre les résultats d'un contrôle effectué avec un traducteur conçu pour focaliser en ondes longitudinales réfractées à $45^{\circ}$. Les acquisitions ont été effectuées sur un bloc de $37,5 \mathrm{~mm}$ d'épaisseur contenant un défaut plan vertical de $15 \mathrm{~mm}$ de hauteur, 
débouchant sur le fond. La taille du défaut est grande devant le diamètre de la tache focale, qui est d'environ $5 \mathrm{~mm}$ (mesuré à $-6 \mathrm{~dB}$ en émission-réception). Plusieurs échos sont enregistrés: on distingue les échos provenant de la diffraction de bord (LL), les effets de coins en ondes longitudinales (LLL) et en ondes transverses (TTT), ainsi qu'un effet de coin mixte faisant intervenir les deux modes de propagation $($ LLT + LTT). La simulation nécessite deux distributions d'amplitude $p(\tau, z)$ différentes, l'une pour les ondes $\mathrm{L}$ et l'autre pour les ondes $\mathrm{T}$. La comparaison entre résultats expérimentaux et simulations montre que les positions et les amplitudes relatives des échos sont bien reproduites par le modèle simplifié. Ces résultats valident ainsi le traitement de l'interaction faisceau-défaut.

Nous avons également pu constater [3] que ce modèle prédit les déphasages observés sur les formes d'onde associées aux différents échos. Cependant la description simplifiée du champ conduit à des formes d'onde constantes le long des échos. Pour prédire les distorsions du signal observées le long d'un même écho, il est nécessaire de calculer la réponse impulsionnelle du traducteur et d'appliquer l'équation (4).

Les résultats d'un calcul complet prenant en compte la réponse impulsionnelle et l'interaction faisceaudéfaut (équation 4) ont pu être comparés avec des signaux expérimentaux provenant du contrôle d'un tube de faible épaisseur (voir figure (3)). La zone examinée contient une fente débouchante et perpendiculaire à l'axe du tube. Sa profondeur atteint $80 \%$ d'une épaisseur totale de $1,3 \mathrm{~mm}$. Le contrôle est effectué avec un traducteur focalisé, qui génère des ondes transverses réfractées à $45^{\circ}$ dans le tube, pour une fréquence centrale de $15 \mathrm{MHz}$. Le modèle néglige la courbure du tube, cette approximation est justifiée car le diamètre externe du tube est grand (environ $20 \mathrm{~mm}$ ) devant celui du faisceau incident (environ $1 \mathrm{~mm}$ ).

L'effet de coin considéré est celui qui apparaît du coté du ligament (dans cette configuration, des effets de coin sont générés sur les deux extrémités du défaut). Les signaux présentés sur la figure (2) correspondent au même effet de coin pour trois positions voisines du traducteur. En $\mathrm{x}=0$, l'axe du faisceau ultrasonore rencontre le point d'intersection entre la ligne du défaut et le fond, c'est la position centrale de l'écho de coin. Les positions suivantes s'éloignent de ce point. On observe alors la déformation, puis le dédoublement des formes d'onde reçues.

La forme d'onde à l'émission entrée en donnée du calcul est une fonction sinus modulée par une fenêtre de Hanning. Ses caractéristiques (fréquence, largeur) sont directement déduites d'un écho de coin expérimental. Les signaux modélisés sont affichés avec une amplitude normalisée. On constate que les déformations et le dédoublement des signaux sont correctement rendus par le modèle qui reproduit de façon satisfaisante l'évolution de la forme d'onde le long de l'écho.

\section{CONCLUSION}

Un modèle est proposé afin de simuler les échos de coin générés par un défaut plan dans une pièce homogène et isotrope contrôlée en immersion. Ce modèle rend compte correctement de l'amplitude, la position et le temps de vol des échos. Le calcul approché de la réponse impulsionnelle du traducteur après réfraction dans la pièce permet de prédire la forme du signal reçu en fonction du balayage. Les premiers résultats sur l'évolution de la forme d'onde le long de l'écho montrent un bon accord avec l'expérience.

[1] L. Brekhovskikh, dans Waves in layered media, Academic Press, pp279-283 (1980).

[2] Ll. G. Chambers, Wave Motion, 2, 247 (1980).

[3] P.Calmon, O.Roy, dans Review of Progress in QNDE, Vol.13, eds. D.O.Thomson and D.E.Chimenti (Plenum Press, New York, 1993), à paraître.

[4] A.Freedman, J. of Acoust. Soc. Am., 48, 270 (1970). 


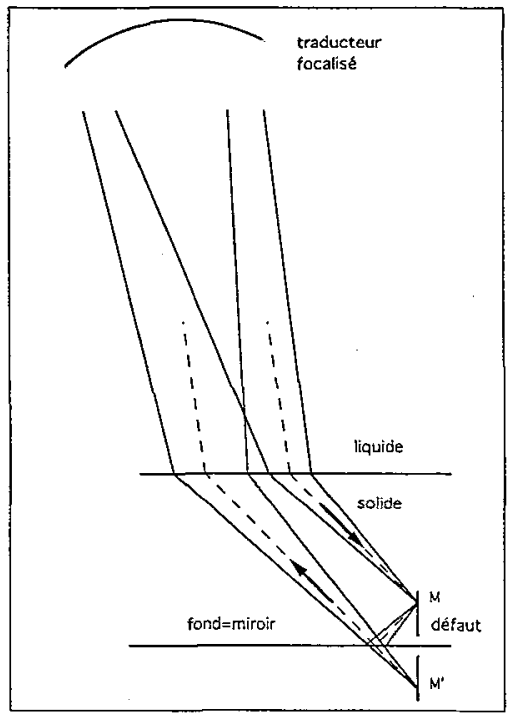

Figure 1: Schéma d'un effet de coin: l'énergie reçue en un point $M$ du défaut semble être reémise à partir d'un point $M^{\prime}$, symétrique de $M$ par rapport au fond.

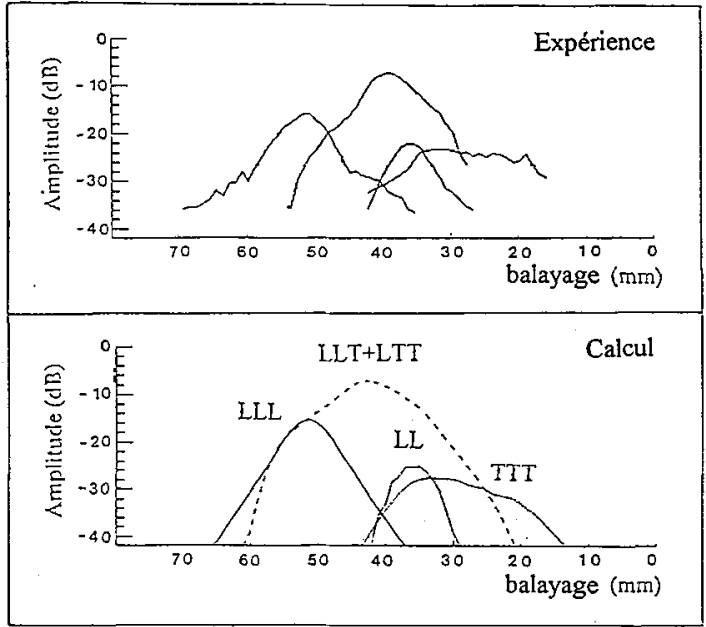

Figure 2: Contrôle effectué avec des ondes $\mathrm{L} 45^{\circ}$ sur un bloc contenant un défaut plan de $15 \mathrm{~mm}$, débouchant sur le fond.

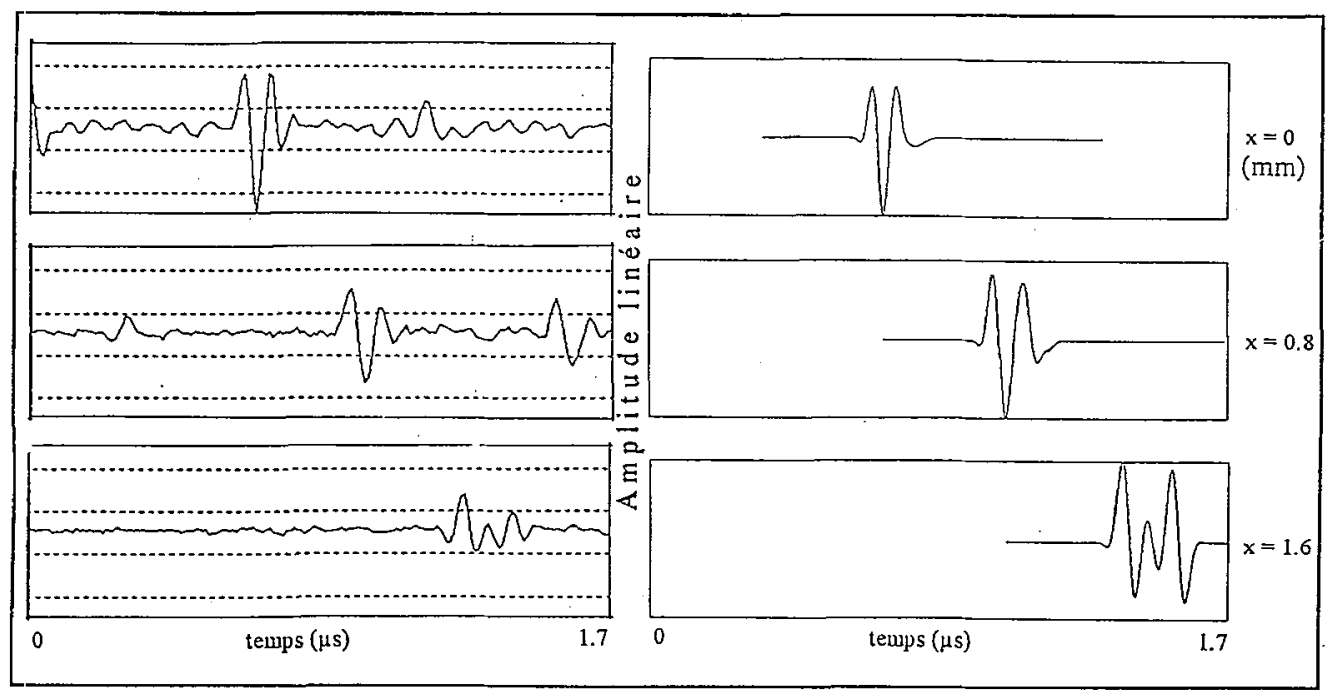

Figure 3: Signaux expérimentaux (à gauche) et simulés (à droite) provenant d'un même écho de coin, obtenus pour trois positions voisines du traducteur. 